\title{
MEANS WITH VALUES IN A BANACH LATTICE
}

\author{
R. RAO CHIVUKULA \\ Department of Mathematics and Statistics \\ University of Nebraska - Lincoln \\ Lincoln, Nebraska 68588 - 0323 \\ and \\ I. RAMABHADRA SARMA \\ Department of Mathematics \\ Nagarjuna University \\ Nagarjunanagar, A.P., 522510 \\ India \\ (Received August 10, 1984 and in revised form September 4, 1986)
}

ABSTRACT. Means, generalized means and invariant means (on a semigroup) with values in a Banach lattice are defined and studied.

KEY WORDS AND PHRASES. Means, amenability, Banach Lattices, Stationary Semigroups. 1980 AMS SUBJECT CLASSIFICATION CODE. 46B30, 46 A40.

1. INTRODUCTION.

Let $S$ be a multiplicative semigroup and $\ell_{\infty}(S)$ the sup-normed Banach space of all real valued bounded functions on $S$. A linear functional $\mathrm{m}$ on $\ell_{\infty}(S)$ is called a mean if $m(e)=1$, where $e$ is the constantly one function, and $m(f) \geq 0$, for every $f$ such that $f(s) \geq 0$, all $s \in S$. A mean $m$ is left invariant if $m\left(\varepsilon^{\prime} f\right)=m(f)$ for all $s \varepsilon S$ and $f \in \ell_{\infty}(S)$ where $\ell$ is the left-translation operator defined by $\left(\ell_{s} f\right)(t)=f(s t)$. The semigroup $S$ is said to be left amenable if $\ell_{\infty}(S)$ admits a left invariant mean. First considered by Von Neumann [1], invariant means have been studied by several authors including Day [2], Dixmier [3] and Mitche11 [4], (a1so Rao [5]).

Means with values in a Banach space were first considered by Dixmier [3] Following his technique Husain and Wong, [6,7] developed a theory of left invariant means with values in $E^{*}$, the dual of a locally convex topological vector space (lctvs) E. Later Husain [8] reformulated and extended the theory to means with values in E. For an lctvs $E$ and a semigroup $S$, he lets $\ell_{\infty}(S, E)$ denote the space of all functions $f: S \rightarrow E$ such that the weak closure $K(f)$ of the convex hull of the range $f(S)$ is weakly compact. With a suitable family of seminorms $\ell_{\infty}(S, E)$ is an lctvs. He defines 
a generalized mean on a subspace $X$ of $\ell_{\infty}(S, E)$ to be a linear operator $M: X \rightarrow E$ such that $M(f) \varepsilon K(f)$ for all $f \varepsilon X$. With these definitions Husain [8] proves that many interesting results from the real valued theory, such as localization principle and Mitchell's theory of stationary semigroups, can be extended to vector valued function spaces.

The real valued function spaces form an interesting subclass of lctvs's, since one can define an order structure induced canonically by the order on reals. In this note we explore the impact of order relation on vector valued means, specifically with values in a Banach lattice. In section 2 we define the spaces $B(S, E)$ and $l_{\infty}(S, E)$ and define means and generalized means. We give a few examples and discuss the compactness of the set of means. In section 3 we present extensions of known results on amenability to the present context, mentioning only those results that are not included in Husain's theory. In the last section we sketch a generalization of Mitchell's theory of stationary semigroups showing that the left amenability of a right stationary semigroup may be obtained in this broader context.

2. COMPACTNESS.

Let $S$ be a multiplicative semigroup, $E$ a Banach lattice with order unit $e$ and $E^{*}$ the normed dual of $\mathrm{E}$. For any $\alpha \varepsilon \mathrm{E}$, the constant function $\alpha: \mathrm{S} \rightarrow \mathrm{E}$ is defined by $\alpha(s)=\alpha$ for all $s \in S$. We define $B(S, E)$ to be the space of all E-valued functions on $S$ whose range is order (and hence norm) bounded in E. (We follow Schaefer [9] for all notions in Banach lattices). With norm defined by $\|f\|=\sup \{\|f(s)\|: s \in S\}, B(S, E)$ is a Banach space; in fact it is a Banach lattice with order unit under the order relation: $f \leq g$ iff $f(s) \leq g(s)$ for all $s \varepsilon S$. It can also be verified that if $E$ is an (order complete) AM-space, so is $B(S, E)$. We now state a proposition (that we need in section 3) which can be proved easily in the standard way.

PROPOSITION 2.1. If $E$ is an AM-space with predual $F$ (an AL-space), then $B(S, E$ ) is isometrically isomorphic to the dual of the Banach space $\ell_{1}(S, F)$ of all absolutely summable F-valued functions on $\mathrm{S}$.

We let $\ell_{\infty}(S, E)$ denote the subspace of $B(S, E)$ consisting of all $f$ such that the weak closure $P(f)$ of the smallest convex sublattice containing the range $f(S)$ is weakly compact. When order intervals in $E$ are weakly compact $B(S, E)=l_{\infty}(S, E)$, (see proof of 2.2 , below). In particular $B(S, R)=l_{\infty}(S)$ where $\mathbb{R}$ denotes the reals. In the sequel we take all subspaces $X$ of $B(S, E)$ to contain the constant functions. Occasionally, $E$ is assumed to be an AM-space; the $K$ in $E=C(K)$ will always be compact Hausdorff. 
PROPOSITION 2.2. Let $X$ be a subspace of $B(S, E)$ and $T: X \rightarrow E$ be linear. Consider the following three conditions:

$$
\begin{aligned}
& M_{1}: \text { For every } f \in X, T(f) \varepsilon P(f) . \\
& M_{2}: \text { If } f(s) \geq 0 \text { for all } s \varepsilon S \text {, then } T(f) \geq 0 \text {; and for every } \alpha \varepsilon E, T(\alpha)=\alpha \text {. } \\
& M_{3}: \text { If } \alpha \leq f(s) \leq \beta \text { for all } s \varepsilon S \text {, then } \alpha \leq T(f) \leq \beta .
\end{aligned}
$$

Then $M_{1} \Longrightarrow M_{2} \Longrightarrow M_{3}$. Further if order intervals in $E$ are weakly compact, then the three conditions are equivalent.

PROOF. It is clear that $M_{1} \longrightarrow M_{2} \Longrightarrow M_{3}$. Now assume that order intervals in $E$ are weakly compact. We show that if $f \varepsilon B(S, E)$ and $\omega$ and $\Omega$ denote respectively the inf and $\sup$ of $\{f(s): s \in S\}$, then $P(f)=[\omega, \Omega]$ the closed interval with bounds $\omega, \Omega$. From this it follows that $M_{3} \Longrightarrow M_{1}$; incidentally, in this case, $B(S, E)=\ell_{\infty}(S, E)$. By theorem 5.10 of $[9],(p .89)$, we have that every element of $E^{*}$ is order continuous and that $E$ is order complete. If $\Gamma$ is a finite subset of $S$, write $\omega_{\Gamma}$ and $\Omega_{\Gamma}$ for the inf and $\sup$ of $\{f(s): s \varepsilon \Gamma\}$. Then $\omega_{\Gamma}+\omega$ and $\Omega_{\Gamma} \uparrow \Omega$. Hence $\omega_{\Gamma} \rightarrow \omega$ and $\Omega_{\Gamma} \rightarrow \Omega$ in the weak topology of $E$. Thus $\omega, \Omega \in P(f)$ and $[\omega, \Omega] \subseteq P(f)$. On the other hand since $P(f)$ is the weak closure of the smallest convex sublattice containing $f(S), P(f) \subseteq$ $[\omega, \Omega]$ and the proposition is proved.

DEFINITION 2.1. Let $X$ be a subspace of $B(S, E)$ and $T: X \rightarrow E$ be linear. Say that $T$ is a generalized mean on $X$ iff $" f \geq 0 \Longrightarrow T(f) \geq 0$ and $T(\alpha)=\alpha$ for every $\alpha \varepsilon$ E". If $X$ is a subspace of $\ell_{\infty}(S, E), T$ is said to be a mean on $X$ iff "T(f) $\varepsilon P(f)$ for all $f \varepsilon X$. We note that by proposition 2.2 , a mean is always a generalized mean and that when order intervals in $\mathrm{E}$ are weakly compact, the two are the same. Thus when $\mathrm{E}=\mathbf{R}$ our definition of a mean agrees with the classical real-valued mean. Also it can be proved (as in the real-valued case) that every generalized mean is continuous and has norm one. We now give some examples.

EXAMPLES 2.1. (a) If $\phi \varepsilon \ell_{1}(\mathrm{~S}), \phi \geq 0$ and $\|\phi\|=1$, then $\phi$ induces a generalized mean $T_{\phi}$ on any subspace $X$ of $B(S, E)$ if we define $T_{\phi}(f)=\sum_{s \varepsilon S} \phi(s) f(s)$. (b) Let $E=C(K), K$ compact Hausdorff. Let $\phi: S \rightarrow E$ be such that (i) $\phi(s) \geq 0$, all $s \varepsilon S$, (ii) $\sum_{s \in S} \phi(s)(t)$ converges uniformly (in $t$ ) to the constantly one function. Then $T_{\phi}$ defined by $T_{\phi}(f)(t)=\sum_{s \varepsilon S} \phi(s)(t) f(s)(t)$ is a generalized mean on any subspace $X$ of $B(S, E)$.

We next consider the compactness of the set of (generalized) means. The real valued means on a subspace $X$ of $\ell_{\infty}(S)$ form a $w^{*}$-compact convex subset of $X^{*}$. Husain 
and Wong $[6,7]$ showed that for an lctvs $E$ and the space $\ell_{\infty}\left(S, E^{*}\right)$ as defined in the introduction, the $E^{*}$-valued means on any subspace $X$ of $\ell_{\infty}\left(S, E^{\star}\right)$ is a compact convex subset of the product space $\left(E_{w}^{*}\right)^{X}$, where $E_{w}^{\star}$ is $E^{*}$ with $w^{*}$-topology. Husain [8] obtained a similar result for E-valued means on subspaces of (his) $\ell_{\infty}(S, E)$. Now turning to our generalized means, it is easy to check that the set of generalized means on a subspace $X$ of $B(S, E)$ is convex. In fact more is true. For if $T_{1}, T_{2}$ are generalized means and $T: X \rightarrow E$ is such that $T_{1} \leq T \leq T_{2}$ then $T$ is also a generalized mean and hence the set $\mathcal{M}(X)$ of generalized means on $X$ is an order interval of the ordered vector space $L^{b}(X, E)$ of all order bounded linear operators from $X$ to $E$. When $E$ is order complete and $X$ is a vector lattice, then $L^{b}(X, E)$ is a vector lattice and $\mathcal{M}(\mathrm{x})$ is a convex sublattice. The following example shows that $\mathcal{M}(\mathrm{x})$ is not compact, in general, even when $\mathrm{E}$ is given the weak topology.

EXAMPLE 2.2. Let $K$ be compact Hausdorff and $S=\{\alpha \varepsilon E:|| \alpha|| \leq 1\}$, the closed unit bal1 in $E=C(K)$. Let $X=B(S, E)$ and $i: S \rightarrow E$ be the identity (inclusion) map [that is, $i(s)=s$ ] and let $E_{w}$ denote $E$ with the weak (that is, $\sigma\left(E, E^{*}\right)$ ) topology. Now if $\Pi_{i}: E_{w}^{X}+E_{w}$ is the (projection) map defined by $\Pi_{i}(\theta)=\theta(i)$, then $\pi_{i}$ is continuous. Hence if $\mathcal{M}(X)$ is a compact subset of $E_{w}^{X}$, then $S=\pi_{i}(\mathcal{M}(X))$ is a compact subset of $E_{w}$ which is not true unless $K$ is a finite set.

In the positive direction, we have the following PROPOSITION 2.3. If order intervals in $E$ are weakly compact then the set of generalized means $\mathcal{M}(X)$ on a subspace $X$ of $B(S, E)=\ell_{\infty}(S, E)$ is a compact subset of $E_{w}^{X}$. PROOF. For each $f \varepsilon X, \mathcal{M}(f)=\{T(f): T \varepsilon \mathcal{M}\}$ is a subset of the order interval $\left[\omega_{f}, \Omega_{f}\right]$ where $\omega_{f}$ and $\Omega_{f}$ are respectively the inf and sup of $\{f(s): s \varepsilon s\}$. Thus $\mathcal{M}(X)$ is a subset of the compact set $\prod_{f \in X}\left[\omega_{f}, \Omega_{f}\right]$, and it is easy to check that $\mathcal{M}(X)$ is closed in $E_{w}^{X}$ and hence compact.

COROLLARY 2.1. If $E=C(K)$ and $E_{p}$ denotes $E$ with the topology of pointwise convergence then the set of generalized means on any subspace $X$ of $B(S, E)$ is a compact subset of $E_{p}^{X}$.

3. MAIN RESULTS.

It is well known that an abelian semigroup $S$ is amenable [2], that is, $\ell_{\infty}(S)$ admits a left invariant mean. This classical result is a consequence of the well known condition of Dixmier $[10, p .4]$. We do not find an analogue of this result in Husain's theory $[6,7,8]$. The richness of the order structure in a Banach lattice enables us to 
obtain this result in the present context. Recall that for $s \in S$ and $f \varepsilon B(S, E)$, $\left(\ell_{s} f\right)(t)=f(s t)$. A subspace $X$ of $B(S, E)$ is said to be left invariant if $\ell_{s}(X) \subseteq X$ for all $\mathrm{s} \varepsilon \mathrm{S}$. A generalized mean $M$ on a left invariant subspace $X$ is said to be

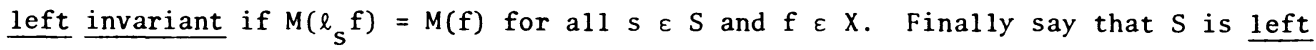

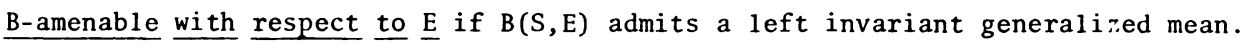
Dually, right invariant generalized means can be defined in a similar way. The following theorem shows that Dixmier's condition is valid for means with values in a Banach lattice.

THEOREM 3.1. Let $\mathrm{E}$ be an order complete Banach lattice with an order unit and $\mathrm{S}$ be a semigroup. A necessary and sufficient condition that $S$ be left $B$-amenable with respect to $E$ is: For all finite sets $s_{1}, s_{2}, \ldots, s_{n}$ in $S$ and $f_{1}, f_{2}, \ldots, f_{n}$ in $B(S, E)$

$$
\inf _{s \in S}\left\{\sum_{i-1}^{n}\left(f_{1}-\ell_{s_{i}} f_{i}\right)(s)\right\} \leq 0
$$

PROOF: Necessity is clear. Assume (3.1) holds and let $X$ denote the subspace of $B(S, E)$ generated by the elements $f-\ell_{s} f$, where $f \varepsilon B(S, E)$ and $s \varepsilon S$. It is clear that for $\phi \varepsilon X, \inf \{\phi(s): s \varepsilon S\} \leq 0 \leq \sup \{\phi(s): s \varepsilon S\}$. Let $p: B(S, E) \rightarrow E$ be defined by $p(f)=\sup \{f(s): s \in S\}$. Clearly $p$ is a seminorm (with values in $E$ ) and $p(\phi) \geq 0$ for a11 $\phi \varepsilon X$. Now by the Hahn-Banach theorem for order complete spaces [12, p.79], there exists a linear map $T: B(S, E) \rightarrow E$ such that $T(f) \leq p(f)$ for all $f \varepsilon B(S, E)$, and $T(\phi)=0$ for all $\phi \varepsilon X$. Since $T$ is linear we have: $-T(\phi) \leq p(-\phi)=\sup \{-\phi(s): S \varepsilon S\}=$ $-\inf \{\phi(s): s \varepsilon S\}$, so that $\inf \{\phi(s): s \varepsilon S\} \leq T(\phi) \leq \sup \{\phi(s): s \varepsilon S\}$. Hence $\mathrm{T}$ is a generalized mean and clearly $\mathrm{T}$ is left invariant.

COROLLARY 3.1. If $\mathrm{E}$ is order complete and $\mathrm{S}$ is abelian then $\mathrm{S}$ is B-amanable with respect to $\mathrm{E}$.

We omit the proof since it is similar to theorem 1.2 .1 of $[10, p .5]$. The evaluation operator $\delta_{s}$ defined by $\delta_{s}(f)=f(s)$ is clearly a generalized mean on any subspace $X$ of $B(S, E)$. When $E=R$, the convex hull of these evaluations is $w^{*}$-dense in the set of means. As in the case of means with values in an lctvs [8], it is not known if the closure of the convex hull of these evaluations is the set of all means on $x$. However, as in the case of means with values in an lctvs, we can prove that means on $X$ can be generated by means on $\ell_{\infty}(S)$ and viceversa. This is the content of the following theorem, whose proof is omitted since it is similar to [8,Thm.6].

THEOREM 3.2. If $X$ is a subspace of $\ell_{\infty}(S, E)$ and $M$ is a mean on $X$, then $M$ induces a mean 
$m$ on $\ell_{\infty}(S)$ satisfying $m\left(x^{*} \circ f\right)=x^{*}(M f)$ for every $f \varepsilon X$ and $x^{*} \varepsilon E^{*}\left[\right.$ here, $\left(x^{*} \circ f\right)(s)=$ $\left.x^{*}(f(s))\right]$. Conversely if $m$ is a mean on $\ell_{\infty}(S)$, then the above equation defines a mean $M$ on $X$. If $m$ is left invariant then so is $M$. Conversely if $M$ is left invariant, then $m$ is left invariant on the subspace $\left\{x^{*} \circ f: x^{*} \varepsilon E^{*}, f \varepsilon X\right\}$ of $\ell_{\infty}(S)$.

An interesting corollary of the above theorem is

COROLLARY 3.2. (Day $[2, p .517]$ ). If $S$ and $T$ are left amenable semigroups then so is their cartesian product $\mathrm{S} \times \mathrm{T}$.

PROOF. Let $\psi$ denote the canonical isometry of $\ell_{\infty}(\mathrm{S} \times \mathrm{T})$ onto $\ell_{\infty}\left(\mathrm{S}, \ell_{\infty}(\mathrm{T})\right)$ and 1 et $\mathrm{m}_{1}, \mathrm{~m}_{2}$ be left invariant means on $\ell_{\infty}(S)$ and $\ell_{\infty}(T)$ respectively. Let $M_{1}$ be the mean on $\ell_{\infty}\left(S, \ell_{\infty}(T)\right)$ induced by $m_{1}$ (by Theorem 3.2) such that $M_{1}(f)(t)=m_{1}(s \rightarrow f(s, t))$. Since $m_{1}$ is left invariant, so is $M_{1}$. Now define $m=m_{2} \circ M_{1} \circ \Psi$. It is not hard to check that $\mathrm{m}$ is a left invariant mean on $\ell_{\infty}(S \times T)$.

4. STATIONARY SEMIGROUPS.

A semigroup $S$ is said to be right stationary if for every $f \in \ell_{\infty}(S)$, the weak* closure $Z(f)$ of the convex hull of the right translates of $f$ has a constant function. Mitchel1 [4] proved that a right stationary semigroup is left amenable. Generalizing this, Husain [8] (and Husain and Wong [6,7]) defined a semigroup $S$ to be right

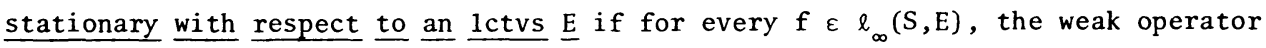
closure of the convex hull of the right translates of $f$ has a constant map. By using an analogue of the localization principle of Granirer and Lau [11], Husain [8] proved that if $S$ is right stationary with respect to $E$, then $\ell_{\infty}(S, E)$ has a left invariant mean. These notions carry cver to the present context of means with values in a Banach lattice yielding similar results. As such we do not present them here and instead, try to enlarge the set $Z$ (f) (of Mitchell and Husain). One natural way seems to be to take $Z(f)$ to be the smallest convex sublattice containing the right translates and closing it in a suitable topology.

Now let $E$ be an order complete AM-space and such that E is the dual of an AL-space $F$. Write $X=B(S, E)$ and $V=l_{1}(S, F)$. By Prop. 3.2 it follows that $X=V^{*}$. Let $Z(f)$ denote $w^{*}$-closure of the smallest convex sublattice in $X$ containing all of the right translates of $f$. We now have

THEOREM 4.1. If for every $f \varepsilon X, Z(f)$ has a constant map $\alpha$, then for each $f \varepsilon X$ and the corresponding $\alpha \varepsilon E$, there exists a generalized mean $M$ on $X$ such that $M(f)=\alpha$.

We present only an outline of the proof of this theorem, since it is similar to the proof of Mitche11's theorem 3.1 [4]. (1) If $f, g \in X$ and $P(f)=$ smallest convex 
sublattice containing all right translates of $f$, then $P(f+g) \subseteq P(f)+P(g)$.

$Z(f)$ is a $w^{*}$-compact order bounded subset of $X$

(3) $Z(f+g) \subset Z(f)+Z(g)$.

(4) The set

$C(f)$ of all values of constant maps in $Z(f)$ is a $w^{*}$-compact subset of $E$ and contains its bounds. (5) If $g \in Z(f)$, then $Z(g) \subseteq Z(f)$. (6) If $S$ is right stationary with respect to $E$ then for every $f, g \in X, C(f+g) \subseteq C(f)+C(g)$. (7) For $f \varepsilon X, l e t$ $p(f)=\sup P(f)$. Then (a) $p(f+g) \leq p(f)+p(g)$, (b) $p(r f)=r p(f)$ for all nonnegative reals $r$ and $(c) p(-f)=-p(f)$. Now let $Y$ be the subspace spanned by any given $f$ in $X$ and define $\theta: Y \rightarrow E$ by $\theta(r f)=r \alpha$. Clearly $\theta$ is linear on $Y$ and $\theta \leq p$. By the Hahn-Banach theorem [12,p.79], $\theta$ extends to a linear map $M: X \rightarrow E$ such that $M \leq p ;$ and $M$ is a generalize mean.

We are unable to show that the $M$ obtained above is left invariant (it seems likely that, in general, it may not be), as is the case in Mitchell's and Husain's theorems. However it is possible to obtain left invariance if we replace $Z(f)$, above, by a smaller set $W(f)$ defined below (but still larger than Husain's $Z(f)$ ). Let $W$ denote the set of all operators $T: X \rightarrow X$ such that (1) ||$T||=1$, (2) $T \geq 0$, (3) $T$ is weak operator continuous, and (4) T commutes with left translations. The set $W$ contains all right translations and is a convex sublattice. Now if for each $f \varepsilon X$, the $W^{\star}$-closure of $W(f)=\{T f: T \varepsilon W\}$ has a constant function, then $M$ is left invariant. (The proof is similar to the one outlined above).

NOTE. Some of the results reported in this paper are based on results included in a thesis for the Ph.D. at the University of Nebraska-Lincoln.

\section{REFERENCES}

1. VON NEUMANN, J. Zur al1gemeinen theorie des Masses, Fund. Math 13(1929), 73-116.

2. DAY, M.M. Amenable Semigroups, Illinois J. Math, 1(1957), 509-554.

3. DIXMIER J., Les moyennes invariantes dans les semigroups et leurs applications, Acta Sci Math (Szeged), 12(1950), 213-227.

4. MITCHELL, T. Constant functions and left invariant means on Semigroups, Trans. Amer. Math. Soc. $119(1965), 244-261$

5. RAO, C.R. (= R.RAO CHIVUKULA) Invariant means on spaces of continuous or measurable functions, Trans. Amer. Math. Soc. 114(1965), 187-196.

6. HUSAIN, T. and WONG, J.C.S. Invariant means on vector valued functions I, Ann. Scuola Norm. Sup. Pisa 27 (1973), 717-729.

7. HUSAIN, T. and WONG, J.C.S. Invariant means on vector valued functions II, Ann. Scuola Norm. Sup. Pisa 27 (1973), 729-742.

8. HUSAIN, T. Amenability of locally compact groups and vector valued function spaces, Symposia Mathematica 16 (1975), 417-431.

9. SCHAEFER, H.H. Banach lattices and positive operators, Springer-Verlag, New York 1974. 
10. GREENLEAF, F.P. Invariant means on topological groups and their applications, Van Nostrant Math. Studies No. 16, New York, 1969.

11. GRAINIRER, E. and LAU, A.T. Invariant means on locally compact groups, III. J. Math. 15(1971), 249-257.

12. PERESSINI, A.L. Ordered topological vector spaces, Harper and Row, New York 1967. 


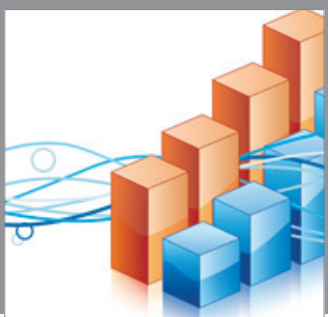

Advances in

Operations Research

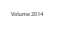

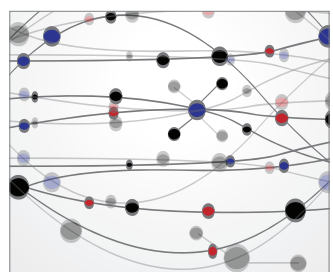

\section{The Scientific} World Journal
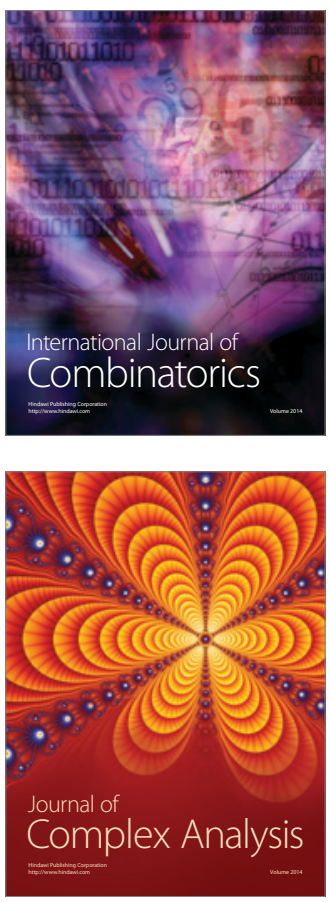

International Journal of

Mathematics and

Mathematical

Sciences
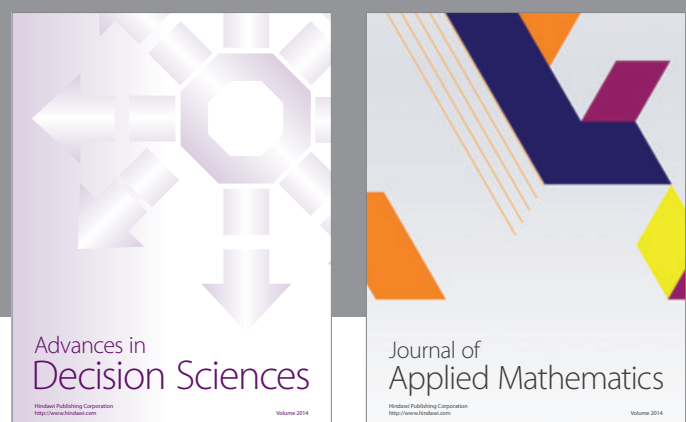

Journal of

Applied Mathematics
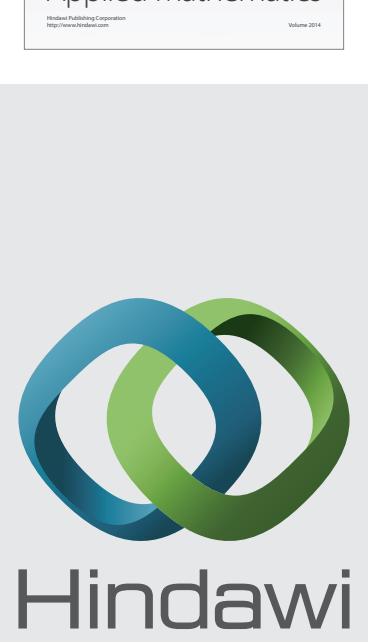

Submit your manuscripts at http://www.hindawi.com
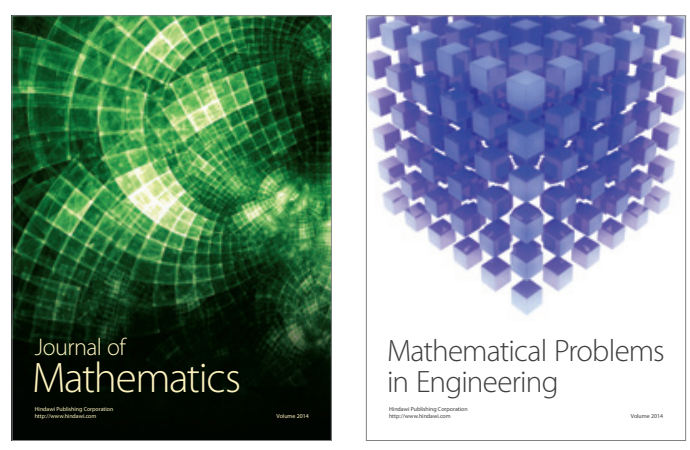

Mathematical Problems in Engineering
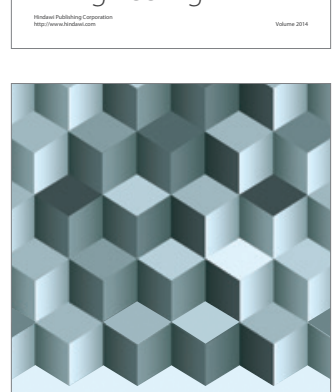

Journal of

Function Spaces
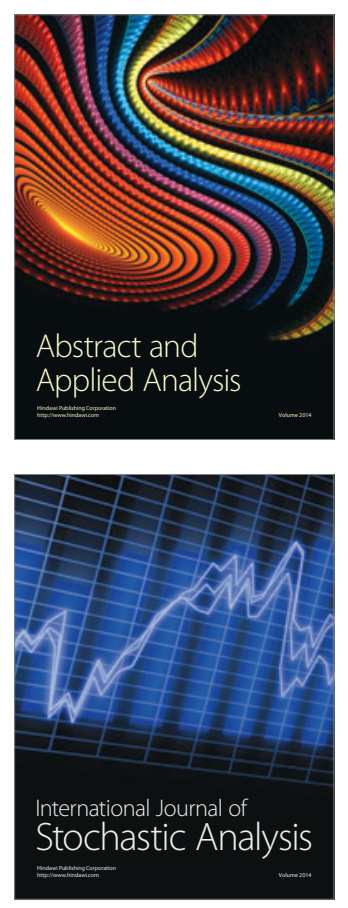

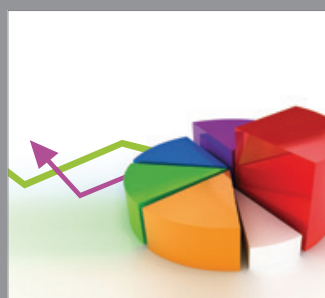

ournal of

Probability and Statistics

Promensencen
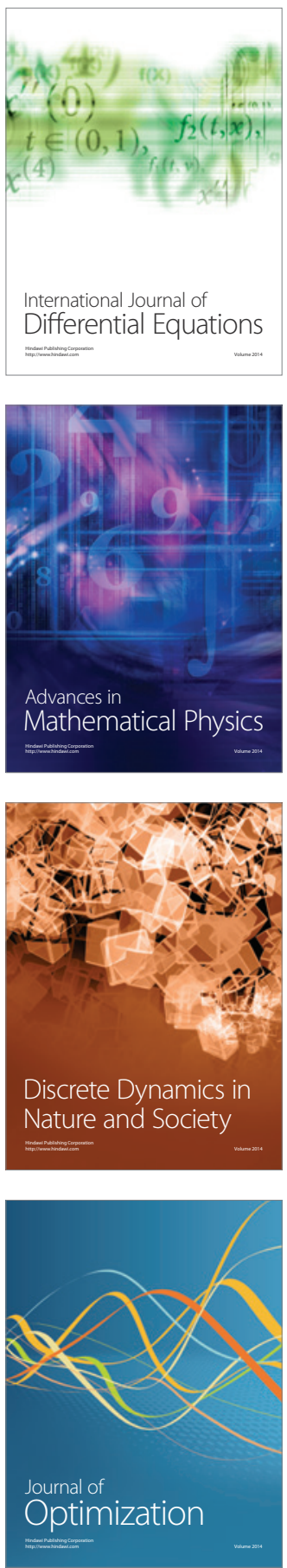